\title{
XXI.
}

\section{Gedächtniss - theoretische Untersuchungen und mnemotechnische Spielereien im Altertum.}

\author{
Von \\ Dr. Bergemann - Jena.
}

2.

Die von Aristoteles begründete wissenschaftliche Psychologie fand in der Folgezeit kaum irgend welche Weiterbildung. Des Neuen war so viel geboten worden; es galt sich zu orientieren; zu sammeln und zu sichten. Dazu kam, dass die schöpferische Kraft der Hellenen erlahmt war; und wenn sie sich doch hin und. wieder nochmals regte, so geschah dies fast ausschliesslich auf praktischem Gebiete: das Problem der Weltbefreiung und der Welterlösung hatte das der Erkenntniss abgelöst. Hatte doch die gebildete Welt den Halt der Religion verloren, den des Staates aufgeben müssen: so suchte man denselben in einer auf die Lebensweisheit gerichteten Philosophie und schliesslich in einer allen realen Boden unter den Füssen verlierenden Mystik. Im grossen und ganzen kann aber, wie angedeutet, die Zeit nach Aristoteles charakterisiert werden als die Epoche der Gelehrsamkeit, der Polyhistorie, der Schematisierung und Systematisierung, mit einem Worte der Aus- wenn auch kaum der Weiterbildung der durch Aristoteles begründeten Special-Wissenschaften. - An die Erörterung psychologischer Fragen trat man in der Folgezeit aus einem doppelten 
Grunde heran: die einen wurden 'dazu geführt durch praktische Erwägungen, die anderen veranlasste dazu das speculative Interesse. Dies letztere war der Fall bei den stoischen, epikureischen und neuplatonischen Philosophen; das erstere gilt hinsichtlich der Aerzte und der Professoren der Weltweisheit und Beredsamkeit. Für den vorliegenden Fall, bei dem es sich ja nur um einen kleinen Ausschnitt aus dem psychologischen Forschungsgebiete handelt, ist allerdings die Ausbeute nicht sehr gross. Von jener Gruppe kommen für uns nur die Neuplatoniker in Betracht, von dieser die Ausbildner jener Kunst, die auch heutzutage trotz vielfachen und energischen Widerspruchs von competenter Seite noch nicht allen Kredit verloren hat, der Mnemonik. Wenn ich oben nur die Neuplatoniker erwähnte, so will ich damit natürlich nicht sagen, dass sich bei den anderen gar keine auf das Gedächtniss Bezug nehmende Erörterungen fänden, sondern nur dass dieselben durchaus belanglos sind. So stossen wir bei den stoischen Philosophen bis auf Seneca und Epiktet herab auf Bemerkungen über das Gedächtniss, von denen aber höchstens das eine hervorhebenswert erscheint, nämlich die Behauptung, dass nur Einwirkungen von starkem Tonus oder oft wiederholte im Gedächtnisse haften bleiben (vgl. L. Stein: Erkenntnisstheorie der Stoa. Berlin 1888, S. 150ff.). Eine Behauptung, die - wie die Erfahrung lehrt - durchaus nicht der Wirklichkeit entspricht, wenigstens nicht in dieser Allgemeinheit: wohl haften dergleichen Reize besser im Gedächtnisse als solche, bei denen diese Bedingungen nicht erfüllt sind - aber auch diese geben nicht verloren, hinterlassen mehr als momentane Eindrücke, woron man sich leicht in Zuständen nervöser Ueberreiztheit oder bloss hochgradiger Erregung überzeugen kann. Von Epiktet ist noch die Bemerkung erwähnenswert, dass das Gedächt-

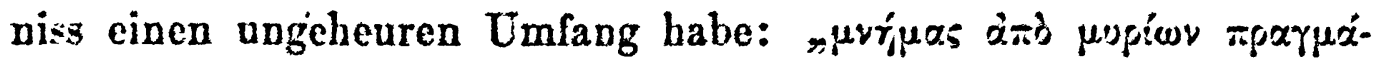

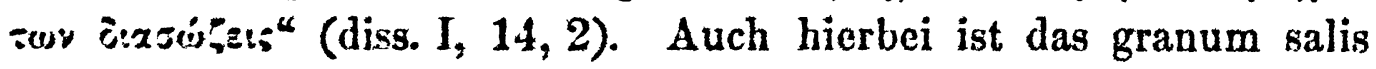
nicht zu übersehen. Gewiss ist der Cmfang des Gedächtnisses sehr gross, aber bei den verschiedenen Arten des Gedächtnisses immer den normalen Durchschnitt natürlich vorausgesetzt durchaus nicht in der nämlichen Ausdehnung; namentlich hinsichtlich der Sprachfertigkeit muss man sich vor einer Ueber- 
schïtzung des Gedächtnisses hüten. Auch ist nicht $\mathrm{zu}$ vergessen, lass, so gross der Umfang des Gedächtnisses auch ist, in einem gegebonon Augenblicke man immer nur an weniges sich zu erinncrn vermag. -

Unter den Neuplatonikern erweckt unser Interesse vornehmlich Plotin (205-270 n. Chr. G.). Im: Gegensatze zu Aristoteles, der, wie ich erwähnt, den Dualismus überhaupt wie im besonderen auf anthropologischem Gebiete zu überwinden versucht hatte, halten Plotin und die Neuplatoniker an demselben ganz entschieden fest, noch consequenter dabei verfahrend als ihr geistiger Vater, als Platon. Indem sie die ja durchaus dualistische Grundlage seiner Philosophie zu der ihrigen, zum Ausgangspunkte ihres Denkens machten, führten sie jenes phantastische System auf, das man als den poetischesten und erhabensten Lobgesang der dualistischen Weltanschauung bezeichnen kann. - Der Mensch ist dem Plotin ein Doppelwesen, bestehend aus Seele und Leib; jener kommt gegenüber diesem unbedingte Selbständigkeit und Substanzialität zu: den psychischen Phänomenen :entspricht kein physischer Parallel-Vorgang. Ein materielles Substrat der Empfindung giebt es nicht; denn dagegen spreche die Thatsache der Erinnerung, da ja nach der materialistischen Anschauung „immer

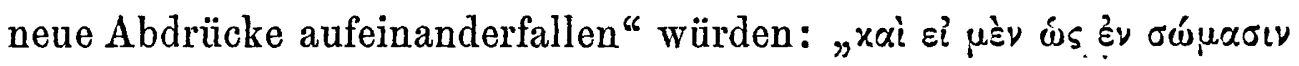

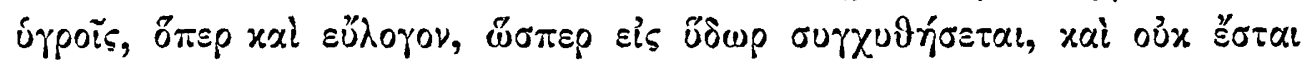

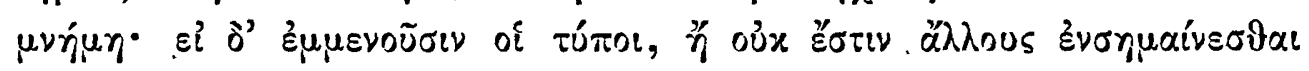

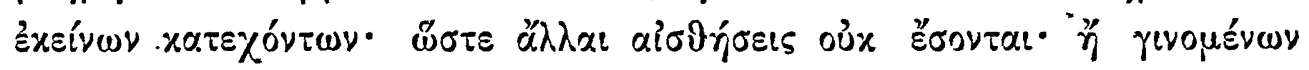

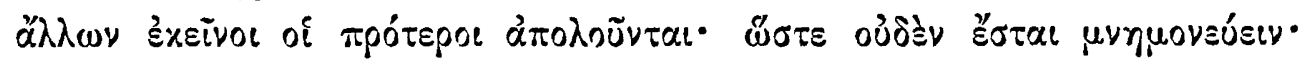

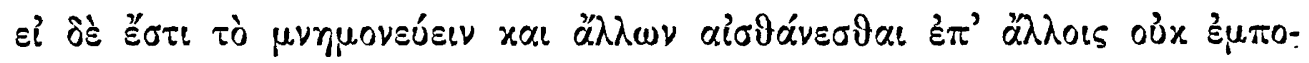

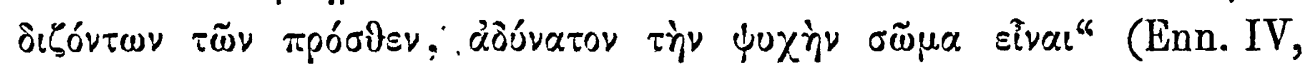
7,6). - Daraus ist ersichtlich, dass wir bei. Plotin auf keine psycho - physische Gedächtnisstheorie, wie bei Aristoteles, stossen werden. Ist für unseren Philosophen doch das Gehirn nicht Sitz der Seele, sondern nur der Anfangspunkt alles Strebens (Enn. IV, 2, 23), wie in der Leber der Anfang der Begierde, im Herzen der des Gefühls zu suchen,.ist., „Die Sinne sind nichts als. das nach aussen gewandte innere Schauen der. Seele" (Enn. IV, 5, 2). Die Erinnerung ist für Plotin eine rein seelische Funktion, in noch 
höherem Grade als die Empfindung. Das Gedüchtniss ist eine Kraft der Seele, ist seelische Activität, kein blosses passives Hinnehmen:

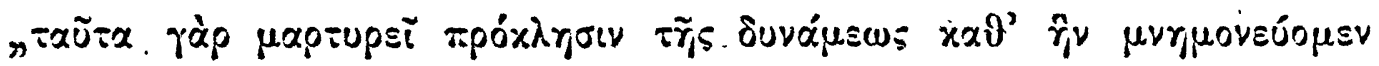
$\tau \tilde{r}_{5}=40 \times \tilde{r}_{s} s^{\prime \prime}$ (Enn. IV , 6, 3). Unter den Gründen, durch welche er dieso Behauptung stützt; hebe ich folgenden besonders hervor: Wäre das Gedïchtnis bloss dio Vorratskammer für äussere Eindrücke, so dürfte es nicht schwïcher werden durch die Fülle solcher ${ }^{18}$ ). Da dies aber der Fall ist, so kann es eben nichts anderes sein als eine Kraft, welche durch vermehrte Arbeitsleistung sehr schnell abgenutzt, verbraucht wird. Auch sei, meint Plotin, wenn es anders wäre, die Thatsache des selbständigen Besinnens unerklïrlich ${ }^{19}$ ). - Freilich ist der Leib nicht ohno jeden Einfluss auf diese Verhältnisse; aber seine Wirksamkeit ist eine ganz negative: er bedingt nicht positiv Erinnerung, sondern infolge der Veränderlichkeit, der er unterworfen ist, greift or nur hindernd ein, bewirkt er das Vergessen. Wie dies jedoch möglich sei, vermag Plotin nicht im geringsten überzeugend darzulegen. Secle und Körper haben ja seiner Ansicht zufolge gar nichts miteinander zu thun, ihrer völligen Wesensverschiedenheit halber ist eine wechselseitige Einwirkung ganz ausgeschlossen; jedes geht glcichsam seinen eigenen Weg. Plotin sagt, die Seele sei im Leibe nicht, wie man so oft hören könne, wie in einem Gefïsse oder Substrat, wie der Teil im Ganzen, die Form in der Materie, der Steuermann im Schiffe, die Technik in den Werkzeugen, sondern auf das Verhältniss der Seele zum Leibe passe nur die Analogie des Verbaltens

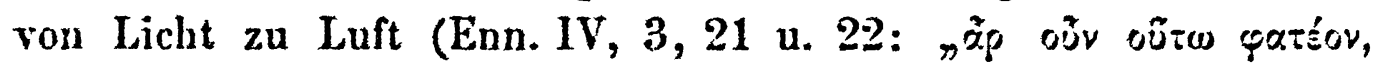

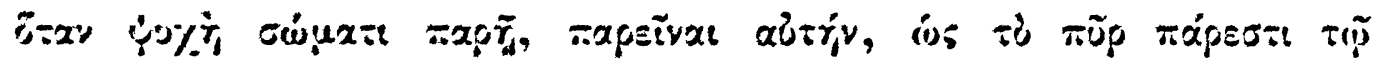

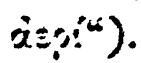

Die Thatsache, dass zwischen Seele und Leib eine gewisse Wechselwirkung stattfindet, konnte jedoch Plotin nicht gänzlich übersehen, sie nötigte ihn zu der allerdings sehr mystisch klingenden Concession, der Körper habe gleichsam einen Schatten der

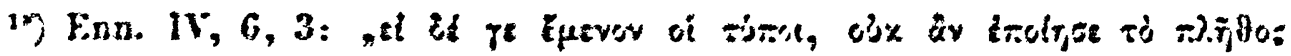

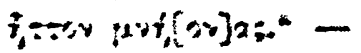

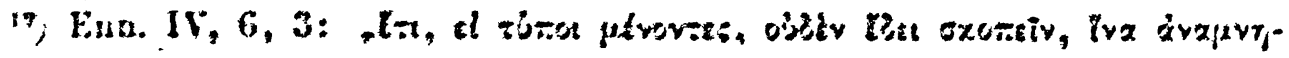
escisiz:- -
} 
Secle, "wodurch' zwischen beiden gleichartige Affection hergestellt werde" (Enn. IV , 5, 1) ${ }^{20}$ ). Aber welchen Causal-Zusammenhang man im einzelnen zwischen diesem halben der monistischen Auffassung gemachten Zugeständnisse und der Behauptung, dass der Leib das Vergessen bedinge, besteht, wird nirgends gesagt, wenn auch zugegeben werden muss, dass durch jene Einräumung diese Einwirkung nicht mehr gar so ungereimt erscheint. -

Die alte Lehre von den Seelenteilen aufnehmend ${ }^{21}$ ) und umdeutend unterscheidet Plotin einen unteren und oberen Teil der Seele, die aber innerhalb des Lebensprocesses ”eine intensive Einheit bilden" und dies wieder „unbeschadet der Trennbarkeit des besseren Teiles von dem schlechteren" (Enn. IV, 3, 31; I, 1,12): die Seele ist in allen ihren Akten ganz und ungeteilt (Enn. IV, 2, 2), und immer wirkt die ganze Seele, wenn auch in verschiedener Weise. Jeder der beiden Seelenteile hat nun seine besonderen Erinnerungen; beim Abscheiden aus 'der' Leiblichkeit beharren nur die des oberen (Enn. IV, 3, 30.32). 'In dem unteren Seelenteil ist der Sitz unserer Erinnerungen äusserer Thatsachen und Begebenheiten, der gesamten Schicksale unseres irdischen Lebens ${ }^{22}$ ). Vermittelt wird diese Erinnerung durch das

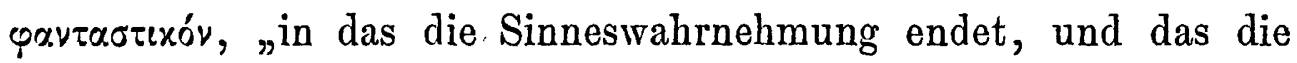
Anschauungen festhält". - Auch dem oberen Seelenteile kommt, wie schon gesagt, Gedächtnis zu und zwar dauerndes, weil es sich - nur hier um höhere, wahrhaft wertvolle Erinnerungen handelt. Denn die denkende Seele, d. i. eben der obere Seelenteil, „behält nur die edleren und bedeutungsvolleren Verhältnisse der Dinge, die mit dem Geistigen in Verbindung stehen, und behält andererseits Gedanken kraft jener selbstbeschauenden Versinnlichung, die. geistige Verknüpfung durch äussere Merkmale einprägt": Sie sitzt gleichsam zu Gericht über die Erinnerungsbilder des unteren

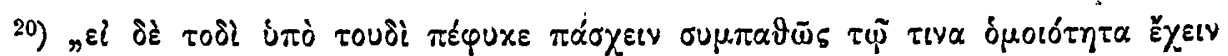

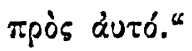

21) Diese Lehre ist' namentlich von Platon ausgebildet worlen'vgl. Zeller, Philos. d. Griechen II, 1. (4. Aufl.) S. $843 \mathrm{ff}$.

22) Vgl. auch Kirchner, Philosophie des' Plotin. Halle 1854. S. $123 \mathrm{ff}$. : „Psychologie des Plotin“; besonders S. $132 \mathrm{ff}$. - 
Seelenteiles, der sinnlichon Seele, sichtet dieselben und ordnet sich ein, was davon wertvoll ist. Ja, je gebildeter die Seele ist,

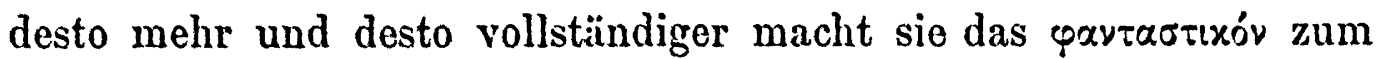
„Organe der höheren Erinnerungen". Auch können beide Seelenteile von einer und derselben Erinnerung bewegt werden: dann ist das Produkt ein ớvizo $\mu x$, ein gemeinsames Bild. Gehen dagegen die beiden Erinnerungen, die beiden $\mu \nu r_{1}^{\prime} \mu \alpha \iota$, auseinander, dann muss eine von beiden weichen. -

Es ist klar, dass Plotin ganz auf der Grundlage der platonischen spekulativen Psychologie weiter gebaut, bezw. sich damit begnügt hat, die bei dem Meister nur vereinzelt auftretenden diesbezüglichen Bemerkungen im idealistischen Sinne weiter auszuführen und auszudeuten. Um die empirische Forschung kümmert er sich so gut wie gar nicht: alle seine Erwägungen stehen im Dienste seiner Metaphysik und der aus dieser sich ergebenden praktischen Consequenzen. Was ihm nicht in seinen metaphysischen Fram passt, ignorirt er. Statt eines Fortschrittes gewahren wir daher, Aristoteles gegenüber, einen offenbaren Rückschritt bei ihm: von einer rein psychologischen Gedächtnisstheorie ist gar keine Rede, noch weniger als selbst bei Platon. Und was von ihm gilt auch hinsichtlich der ganzen neuplatonischen Schule. -

Das Resultat aller bisherigen Ausführungen ist also dies, dass wir aus dem Altertume nur eine einzige.Gedächtniss - Theorie, die trotz vieler Nängel wirklich diesen Namen verdient, besitzen, nänlich die aristotelische; und dies kann uns kaum Wunder nehmen bei einem Problem, das zu den schwierigsten und complicirtesten in der Psychologie gehört, wenn man bedenkt, dass den Alten noch gar sehr die Fähigkeit der ruhigen Selbstbeobachtung abging, ihr ganzes Denken - auch bei den aufgeklärtesten und freiesten Köpfen - durch mythischo Vorstellungen beeinflusst war und ihnen zudem noch das psychologische (und physiologische) Experiment fehlte, wodurch ja erst in der neuesten Zeit mehr Klarheit in die psychologische Forschung gekommen ist, die Psychologie erst jetzt eine eigentliche Wissenschaft zu sein angefangen hat. Ausserdem fühte die Differenzicrung der Wissenschafteu und die Ilochschätzungr encyclopädischen Wissens in der 
Zeit nach Aristoteles von der. Reflexion über das eigentliche Gedïchtniss - Problem ab und ward vielmehr darauf gerichtet, Mittel und Wege zu finden, die Einprägung ins Gedächtniss und das Behalten des Eingeprägten zu erleichtern, dabei teils das Bedürfniss des Rhetors teils dasjenige des gelehrten Polyhistors berücksichtigend: es war die Blütezeit der Mnemotechnik, die durchaus keine moderne Erfindung, sondern eine schon recht alte Spielerei ist. Wenn man bedenkt, dass bis auf Augustus es kein Redner gewagt haitte, mit einem Notizen - Zettel vor das Publikum zu treten, so wird man sich nicht wundern, dass man schon frühzeitig auf mancherlei kleine Kunstgriffe verfiel, um das Gedächtniss. zu unterstützen. Als. Erfinder der Mnemonik galt den Alten ziemlìch allgemein der Dichter Simonides (ca. 559-469 v. Chr. Geb.), jedenfalls im Hinblick auf den Umstand, dass dieser in einem Epigramm sich seiner im 80. Lebensjahre noch ganz ungeschwächten Gedächtnisskraft rühmt ${ }^{23}$ ). 'Beweise für oder gegen diese Annahme lassen sich natürlich nicht beibringen; jedoch ist es wahrscheinlicher, dass, was Morgenstern (Comment. 'de arte veterum mnemonica. Dorpat 1835 S. IV ff.) vermutet, die Mnemonik von einem der Sophisten im Zeitalter des Sokrates ersonnen und nur, um ihr besseren Eingang zu verschaffen, auf den berühmten Lyriker zurückgeführt worden sei, der sich, wie gesagt, noch im spätesten Alter seiner vollen Gedächtnisskraft -rühmen konnte. Uebrigens berichtet Diogenes Laertius auch von Pythagoras, dass er sëinen Schülern Gedächtniss - Uebungen empfohlen habe (Vitae phliosophorum. Graece et latine, ed. Cobet. Paris 1850 S. 510). Wie dem nun auch sein möge, sicher ist, dass, es zur Zeit Ciceros bereits eine ganze mnemotechnische Litteratur gab (vgl.- Cornifici Rhetoricorum ad C. Herennium 1. III. c. 23); Theodektes Phaselita, ein Schüler des Platon, Isokrates und Aristoteles, hatte den Reigen eröffnet, das bereits Vorhandene und Ueberkommene systematisierend und in usum oratoris weiterbildend, und in der Folgezeit, gegen Ende des zweiten vorchristlichen Jahrhunderts, ist Charmadas zu erwähnen als einer, der diesem Litteratur-Zweige seine

23) Vgl. über ihn Suidae Lexikon. Graece et latine. ed. Bernhardy, II. Bd. Halle und Braunschweig 1853. S. $756 \mathrm{ff}$. 
Aufmerksamkeit widmete (vgl. Plinius, hist. nat. VII, 24). - Anfänglich war die Mnemonik der Alten noch ziemlich einfach, ein Complex äusserlicher Mittel, wodurch man das Aufzufassende in gewissen räumlichen Begrenzungen und bildlichen Anschauungen der Einbildungskraft nahe rückte und fassbar machte: man dachte sich grosse Vorstellungsmassen, um sie sich zu merken, örtlich in einer Stadt oder in mehreren Städten und innerhalb dieser wieder in Häusern und Zimmern von bestimmter Anzahl verteilt. Frei von Künstelei ist die Sache natürlich nicht, aber sie wurde durch die fortgehende litterarische Bearbeitung und Systematisierung noch immer complicierter: so z. B. bei Cicero und schon früher bei dem nincerto auctori rhetoricorum ad Herennium". Dieser ist nach Schütz, dem auch Bonnell beistimmt ${ }^{24}$ ), M. Antonius Gripho, ein etwas ālterer Zeitgenosse Ciceros, welcher selbst, als er bereits die Praetur bekleidete, jenes Schule besucht haben soll (Macrob. Sat. III, 12,8): ein berühmter Lehrer der Grammatik und Rhetorik zu Rom (Suet., de ill. gramm. 7). Weniger wahrscheinlich ist Heusdes Vermutung, dass Aelius Stilo jener „incertus auctor" sei. Dieser Rhetor nun (vgl. Rhetor. ad C. Heren. l. III, c. XVI ff.) unterscheidet ein natürliches und ein künstliches Gedüchtniss: „sunt duae memoriae", sagt er, „una naturalis, altera artificiosa; naturalis est ea, quae nostris animis insita est et simul cum cogitatione nata. Artificiosa est ea, quam confirmat inductio quaedam et ratio praeceptionis". Ueber dieses letztere nun will er sprechen, „quae constat ex locis et imaginibus". „Locos" nennt or „qui breviter, perfecte, insignite aut natura aut manu sunt absoluti, ut eos facile naturali memoria comprehendere et amplecti queamus, ut aedes, intercolumnium, angulum, fornicem et alia, quae his similia sunt." Die ,imagines ${ }^{\alpha}$ sind ${ }_{n}$ formae quaedam ot notae et simulacra “ desjenigen nämlich, dessen wir uns erinnern wollen. Wenn wir uns z. B. an Pferde, Löwen, Adler erinnern wollen, so müssen wir "imagines eorum locis certis conlocare". Wollen wir viel in unserem Gedächtnisse festhalten, so müssen wir multos locos nobis comparare, ut in multis locis multos

27) Vgl. De arte memoriae commentatio historica. Programm des Berliner Friedrichs-Gywuasiuers. Berlin 1838. S.9. 
imagines conlocare possimus". Die "loci“ gleichen der Wachstafel, die „imagines“ den eingeritzten Buchstaben. - Die loci müssen nun nach einer gewissen Ordnung gewählt werden, sie müssen auch „forma atque natura" von einander verschieden sein. Ferner dürfen sie nicht zu gross und nicht zu klein, nicht zu nahe bei und nicht zu weit von einander entfernt sein: „intervalla locorum mediocria placet esse, fere paulo plus aut minus pedum trinum". - Die „imagines“ sind doppelter Art, nämlich „unae rerum, alterae verborum. Illae exprimuntur, cum summatione ipsorum negotiorum similitudines comparamus; hae constituntur; cum unius cuiusque nominis et vocabuli memoria similitudine notatur." Das Auffinden von Aehnlichkeiten an Dingen und Geschehnissen und demgemäss das Behalten solcher macht nur "geringe Schwierigkeiten; es kommt dabei oft nur auf ein einziges Merkmal an. Schwieriger ist die Sache dann, wenn es gilt, Worte dem Gedächtnisse einzuprägen. Wie man dabei zu verfahren hat, zeigt er an folgendem Beispiele (a. a. 0. c. 21): es soll der Vers

$$
\text { "Iam domutionem reges Atridae parant" }
$$

gemerkt werden. Er sagt darüber: „In uno loco constituere oportet manus ad coelum tollentem Domitium, cum a regibus Marciis locis caedatur; hoc erit "jam domutionem reges“. In altero loco Aesopum et librum subornari ut ad Iphigeniam in Agamemnonum et Menelaum ${ }^{25}$ ); hoc erit "Atridae parant"; hoc modo omnia verba erunt . expressa". -

Eng an den „incertus auctor" schliesst sich Cicero. (106-43 v. Chr. Geb.) an, der auch ein begeisterter Lobredner der Mnemonik ist. Das Gedächtniss, sagt er (de inv. I, 1) ist eine „firma animi verum - et verborum perceptio“, also Activität, eine Kraft der Seele, keine psychophysische Funktion. Es ist von höchster Wichtigkeit für alle Menschen, besonders auch für den Redner; es ist ein "thesaurus omnium rerum", es muss zum Wächter gesetzt werden

25) Der Text ist hier arg verstümmelt; ihn richtig zu stellen haben viele versucht. Vgl. Kaysers Bemerkungen zu seiner Ausgabe der "Cornifici Rhetoricorum ad C. Herennium Libri IV“. Leipzig 1854. S. 280. 115,1.' - Vgl. auch Bonnel a. a. 0. S. 11. Anmerkung 1.:- 
„inventis cogitatisque rebus et verbis“, sonst „omnia, etiam si praeclarissima fuerint in oratore, peritura" (de oratore $I, 5,18)^{26}$ ). Daher preist èr den Erfinder der Kunst, vermöge welcher man, wenn man sich von Natur keines so ausgezeichneten Gedächtnisses wie etwa Themistokles erfreut, dasselbo unterstützen kann; natürlich aber sei die memoria naturalis die Hauptsache. Ausserordentlich wichtig, meint er, sei es, abstrakte Begriffe, Gedankendinge sich unter dem Bilde eines sinnlichen und zwar sichtbaren Gegenstandes einzuprägen, weil das sinnlich Wahrnehmbare, besonders. das dem Gesichtssinne zugängliche, weit besser im Gedächtnisse hafte; denn ,acerrimus ex omnibus nostris sensibus est sensus videndi; facillime animo teneri potest ea, quae perciperentur auribus aut cogitatione, si etiam oculorum commendatione animis traderentur $^{\star}$ (de orat. II, 87, 357). - Näher geht jedoch Cicero nicht auf die Erörterung der mnemotechnischen Hilfsmittel ein; er erklärt sich eben, wic schon gesagt, mit den vorhandenen einverstanden. Nur hinsichtlich der Einprägung von Worten macht er noch folgende Vorschläge. Mit Bezug auf die Wortbilder, die ,imagines verborum ${ }^{27}$ ), sei von Wichtigkeit die Etymologie (dic Wortbildung im weiteren Sinne): „dadurch, dass man ähnliche Worte bildet durch Umwandlung und Beugung der Eudsilben (Declination), oder dass man ihre Bedeutung von der species auf das genus übertrïgt und durch das Bild eines Wortes einen ganzen Gedanken darstellt" (vermittelst der symbolischen Ausdrucksweise - vergl. de orat. II, 87,358 und auch Top. 8, 35). Der Redner soll wie ein tüchtiger Maler, der auf seinen Gemälden die Perspective und die Distanzen durch die verschiedene Grösse und Anlage der Figuren kenntlich macht, durch die verschieden

50) Vgl. such Or. part. 1,4; 7,26 - Brut. 61,219 - de opt. gen. orat. 2,3 : sed carum omnium rerum ut aedificiorum memoria est quasi fundamenturn.*

ii) Schema der wnemotechnischen IIilsmittel nach dem ,incertus auctor und nach Cicero:

loci - $\frac{\begin{array}{c}\text { imagines } \\ \text { rertorum }\end{array}}{\begin{array}{c}\text { imagines } \\ \text { rerum. }\end{array}}$


gestalteten Wortbilder, durch Wörter desselben Etymon, die darán geknüpfte Gedankenlage sich für sein Gedächtniss kennzeichnen. Freilich auf alle die Wörter, die keine eigentlichen Begriffswörter sind, die Conjunctionen, Präpositionen, Interjectionen, kann das eben Gesagte keine Anweridung finden; denn diese „formari similitudine nulla possunt“. Sie muss man sich unter gewissen stereotypischen Zeichen oder Bildern merken: „eorum fingendae nobis sunt imagines, quibus semper ùtamur" (de orat. II, 88, 359). - Nimmt man zu diesen Hilfsmitteln, die der Redner anwenden soll, um Worte seinem Gedächtnisse einzuprägen noch die schon früher erwähnten betreffend die Einprägung von Gedankendingen hinzu und diejenigen, welche das Sachgedächtniss, das nach Cicero für den Redner noch wichtiger ist als das Wortgedächtniss („rerum memoria propria est oratoris, verborum memoria minus nobis est necessaria"), unterstützen sollen - hierbei soll es hauptsächlich auf zweckmässige Stellung der mnemonischen Bilder ankommen, hinter denen wie unter der Maske der Schauspieler der Gedanke verborgen ist - so ergiebt sich ein recht complicirter mnemotechnischer Apparat, ein ungeheuerer Gedächtniss-Ballast, der aber, wie Cicero naiv genug bemerkt, das natürliche Gedächtniss durchaus nicht beeinträchtigen soll. Er ist so verrannt in seine mnemonischen Marotten, dass er alle Einwürfe dagegen, an denen es nicht fehlte, leichter Hand abthun zu können glaubt: „neque verum est, quod ab inertibus dicitur, opprimi memoriam imaginum pondere et obscurari etiam id, quod per se natura tenere potuisset." -

Weit einsichtiger als Cicero in dieser Hinsicht ist Quintilian (38 bezw. 42 - ca. 118 n., Chr. G.); derselbe, hat eine ziemlich geringe Meinung von der Mnemonik. Vom Gedächtniss und seiner Pflege handelt vornehmlich das zweite Capitel des elften Buches seiner "Institutio oratoria“. Das Gedächtniss, sagt er, ist ein Geschenk der Natur; man müsse es jedoch sorgfältig pflegen, um es nach Umfang und Stärke zu erweitern. Namentlich der Redner bedürfe eines guten Gedächtnisses: für ihn besondeis hätten mnemotechnische Hilfsmittel Wert, wenn ihnen ein solcher überhaupt zukäme. Aber dieselben seien viel-zu gekünstelt und statt das 
Gedächtniss zu unterstützen und das Behalten zu erleichtern, belasten sie es noch viel mehr, indem ihm die Lösung einer doppelten Aufgabe aufgebürdet werde. Jedoch verwirft er nicht gänzlich die Mnemonik; denn „multum signa faciunt, et ex alia memoria venit alia". Allerdings wünscht er, dass die mnemonischen Hilfsmittel möglichst einfach und naturgemäss seien; z. B. „ancora si de nave dicendum esset, spirulum, si de proclio". Gegen dergleichen wird sich kaum etwas einwenden lassen; solche kleine GedächtnissStützen gebrauchen wir wohl alle zuweilen - und ganz mit Recht. - Das Wichtigste jedoch, sagt Quintilian sehr richtig, sei Uebung und Anstrengung: „Si quis tamen unam maximamque a me artem memoriae quaerat, exercitatio est et labor: multa ediscere, multa cogitare, et, si fieri potest, quotidie potentissimum est" (a. a. 0. \$ 40). Und zwar schreite man langsam fort; man lerne zunächst nur einige Verse und vermehre ganz allmählich deren Anzahl. Dieses Verfahren wende man auch beim Unterrichte der Knaben an: denn Gedächtniss - Uebungen müssen von Jugend auf angestellt werden. So nur könne man sich ein treues und umfangreiches Gedächtniss erwerben, vorausgesetzt, dass eine gute natürliche Veranlagung vorhanden ist. Bei einem guten natürlichen Gedächtnisse hätten Männer wie Themistokles und Mithridates, Crassus und Cyrus nur durch Uebung, durch eifriges Studium sich den Ruhm erworben, ein ausgezeichnetes Gedächtniss zu besitzen. Die Künste eines Simonides und Hippias, eines Charmadas und Metrodorus seien mehr oder weniger nur unnütze Spiclereien. - Diese Ansicht Quintilians, die auch hculzutage gegenüber den immer und immer wieder auftauchenden mnemotechnischen Künsteleien, die doch auf einer falschen Voraussetzung die Association anlangend beruhen, die herrschende ist, nachdem schon Kant die Yethode des sogenannten judiciösen" Memorierens in seiner Anthropologie , ungereimt und zweckwidrig " genannt hat, wurde jedoch im Altertume nicht so gewürdigt, wie rie es verdient hătte: vielmehr war in der Folgezeit die Mnemonik sehr im Schwange und musste sich noch obendrein, dem damaligen Feschmacke entsprechend, die Verbrämung mit allerhand magischen Elementen gefallen lassen. Ja, sogar eine ${ }_{n} V$ ergessungs- 
wissenschaft", wio Aretin sich. ausdrückt ${ }^{38}$ ), entstand jetzt, noch extravaganter und verrückter als jene ausgeartete Mnemonik. -

Von hervorragenden Männeru, welche im Sinne Quintilians und zwar besonders infolge pädagogischer Erwägungen GedächtnissUebungen empfehlen, will ich zwei nennen, Plutarch und Lucian von Samosata, mit deren Betrachtung ich die vorliegende Arbeit abschliessen möchte. Beide Männer lebten zu einer Zeit, in welcher unter den Begleiterscheinungen einer hochentwickelten Civilisation in höchst bedenklicher Weise sich eine Trennung der Bildung vom Leben bemerklich machte, zu einer Zeit, wo jene bereits ganz den Charakter einer toten Gelehrsamkeit angenommen hatte. Freilich bekämpfen beide die vorhandenen Uebelstände, dringen auf Verbesserung und träumen von einer Wiedergeburt jenes goldenen Zeitalters des griechischen Altertumes; wo der Mensch in allseitiger Entwickelung seines Geistes wie seines Leibes für Staat und Leben kräftig wirkte und thätig schaffte und das freudige Bewusstsein freier Menschlichkeit im schönen Wirkungskreise sich auch frei und schön bethätigte; wohl polemisiert Lucian gegen das Aufhäufen toten Wissensstoffes und persifliert in den „Gedungenen Gelehrten" aufs köstlichste die Lehrer der Jugend - aber dennoch ist er so gut wie der ein Jahrhundert vor ihm lebende Plutarch ${ }^{29}$ ), berühmter Lehrer der Philosophie in Rom; ein Kind der Zeit. Daher, so sehr sie für das althellenische Bildungs- und Erziehungs-Ideal begeistert sind, meinen sie doch, dass ein junger Mensch von guter Herkunft in keiner der encyklischen Wissenschaften unbewandert sein darf. Er muss, sagt Plutarch ausdrücklich in der Schrift "de educatione puerorum ${ }^{30}$ ), eine nach der

28) Aretin, Systemat. Anleitung zur Theorie und Praxis der Mnemonik, nebst Grundlinien zur Geschichte und Kritik dieser Wissenschaft. Sulzbach 1810. S. 530. Cap. VIII: Geschichte der Vergessungswissenschaft...-

29) Lucian 130-200, Plutarch 60-120 n. Chr. Geb.

30) $\mathrm{Ob}$ diese Schrift wirklich von Plutarch herrührt, ist allerdings zweifelhaft. : Die Unechtheit derselben hat namentlich Wyttenbach behauptet und zu beweisen versucht; seinem Urteile schliesst sich auch Volkmann an in seinem Werke: „Leben', Schriften und Philosophie des Plutarch von Chaeronea." Berlin 1869. I. Teil S: 180. Benseler dagegen bezeichnet die Schrift nur als in hohem Grade verdächtig. . Deinhardt hält sie für echt; sie sei, meint er, 
anderen insoweit erlernen, dass er wenigstens einen Vorgeschmack davon bekommt. Und Lucian spricht in den "Liebkosungen" die nämliche Ansicht aus: für den freigeborenen Jüngling sei eine encyklopädische Bildung nötig, sein Geist müsse mit allen Kenntnissen, die einem solchen ziemen, fleissig genährt werden. Da kann es uns dann freilich auch nicht wundern, wenn beide fleissige Gedächtniss-Uebungen empfehlen, indem sie darauf hinweisen, dass „für den Erfolg des Unterrichtes es vor allem wichtig sei, das Gedächtniss zu üben, durch welches der Wissensbesitz gesammelt und aufbewahrt werde." Das Gedächtniss, sagt Plutarch, lässt Wissen entstehen und nährt es. Gedüchtniss-Uebungen müssen eintreten, ob nun die Kinder von Natur ein gutes Gedächtniss haben oder vergesslich sind: „die Fülle ist zu befestigen, der Mangel zu ergänzen, so werden jene, deren Gedächtniss von Natur gut ist, andere, diese, die ein schwaches Gedächtniss haben, sich selbst übertreffen *. Aber nicht nur für die Aneignung des Wissens empfehlen sie Uebung des Gedüchtnisses, sondern dieselbe sei auch erforderlich für dio Geschäfte und das praktische Leben. „Erfahrung macht klug", lesen wir bei Plutarch, „aber die Erfahrungen nützen uns nichts, wenn wir sie vergessen". Wie in der Empfehlung von Gedächtniss-Uebungen überhaupt so stimmen Plutarch und Lucian auch darin mit Quintilian überein, dass dieselben vornehmlich in vielem Auswendiglernen bestehen sollen. - Wirkönnen hier ganz mit den Behauptungen und Forderungen dieser Männer übereinstimmen: es ist sicher, dass das Gedächtniss durch Uebung bildbar und dass namentlich systematisches Auswendiglernen unerlässlich ist. Denn davon hängt zum grossen Teilo das ab, was man Präsenz des Wissens nennt, nämlich die Fähigkeit, im gegebenen Augenblick leicht und sicher zu reproducieren. Was wir nicht reproducieren können ist für unser Wissen verloren; das ad hoc Gelernte verraucht schnell wieder. -

cin Spätling der Plotarchischen Yuse, daber und reil sie für ein grösseres Publikum bestimmt sei und cinen etwas herablassenden Ton anschlage, wio er sich sonst nicht bei Plutarch finde, mache sich ein ziemlich beträchtlicher Atrstand von seinen übrigen Schriften bemerkbar. - 